\title{
Enhancement of Transient Stability Limit and Voltage Regulation with Dynamic Loads Using Robust Excitation Control
}

\begin{abstract}
In stressed power systems with large induction machine component, there exist undamped electromechanical modes and unstable monotonic voltage modes. This article proposes a sequential design of an excitation controller and a power system stabiliser (PSS) to stabilise the system. The operating region, with induction machines in stressed power systems, is often not captured using a linearisation around an operating point, and to alleviate this situation a robust controller is designed which guarantees stable operation in a large region of operation. A minimax linear quadratic Gaussian design is used for the design of the supplementary control to automatic voltage regulators, and a classical PSS structure is used to damp electromechanical oscillations. The novelty of this work is in proposing a method to capture the unmodelled nonlinear dynamics as uncertainty in the design of the robust controller. Tight bounds on the uncertainty are obtained using this method which enables high-performance controllers. An IEEE benchmark test system has been used to demonstrate the performance of the designed controller.
\end{abstract}

Keywords: voltage regulation, transient stability, robust control, uncertainty, nonlinearity

*Corresponding author: Jahangir Hossain, Griffith University, Gold Coast, QLD 4222, Australia, E-mail: j.hossain@griffith.edu.au Apel Mahmud, The University of Melbourne, Melbourne, VIC, Australia, E-mail: mdapel.mahmud@nicta.edu.au

Naruttam K. Roy: E-mail: n.roy@adfa.edu.au, Hemanshu R. Pota:

E-mail: h-pota@adfa.edu.au, UNSW@ADFA, Canberra, ACT, Australia

\section{Introduction}

Modern power systems are operating increasingly closer to their control and operational limits due to the increase in demand for electric energy coupled with economical, technical, and environmental restrictions on their expansion. Demands for large power transactions in an openaccess market can severely stress a system to the edge of its stability limits. When a stressed power system is subjected to small or large disturbances, it exhibits complex dynamic behaviours [1], which results in different forms of system instability [2], among which transient and dynamic voltage stabilities are very important considerations to provide reliable and efficient operation of power systems.

Transient instability, which is triggered by large disturbances [3], is still one of the main stability issues of power systems. Power oscillations of small magnitude and low frequency often persist for long periods of time which limits the power transfer capability of the system. The use of power system stabilisers (PSSs) has become increasingly important to provide improved stabilisation of the system. The PSS has been designed to add damping to the generator rotor oscillations; however, the voltage modes cannot be stabilised using a PSS [4]. Generators equipped with a PSS and an automatic voltage regulator (AVR) can enhance voltage as well as transient stability of power systems. North American Reliability Council and Western Electric Coordinating Council are ruling that machines rated more than $35 \mathrm{MV}$ A or group of machines equal to or more than $75 \mathrm{MV}$ A connected to the transmission grid through one transformer must operate in voltage regulating mode and be equipped with PSSs to improve the transient stability of the system [5].

The reactive power of a system is tightly related to bus voltages throughout the network, and hence it has a significant effect on dynamic voltage stability and collapses. Recently, the problem in voltage instability is likely to increase because of the growing use of dynamic motor loads for air conditioning, heat pumps, refrigeration, and so forth, which consume a large amount of reactive power after a disturbance [6]. The disturbances in power systems may not only cause the loss of synchronisation but also result in short-term voltage dips and sags. This requires the robust controller to have the 
ability to suppress the potential instability due to poorly damped power angle oscillations that can be dangerous for the system stability and to compensate the voltage dips and sags that can damage both utility and customer equipments.

Power systems can be stabilised either by including flexible alternating current transmission systems devices which control the power flow and regulate the voltage level or by controlling the excitation of synchronous generators [7]. As the use of excitation controller is economical, this work is mainly focused on this approach. To improve overall performances of the system, the coordination between PSSs and excitation controllers has been reported in the literature [8-11]. Some of these methods are based on the complex nonlinear simulation, and the others are based on the linearised power system model. Excitation controllers designed based on approximately linearised models depend on a given set of operating conditions and, therefore, may not work properly when the operating conditions change [12].

As the real electric power systems have been experiencing a dramatic change, the application of advanced control techniques in power systems has attracted a great attention in recent years to ensure secure system operation under wide operating ranges [8]. A coordinated AVR and PSS control has been presented for a single machine infinite bus (SIMB) system which allows coordinated trade-off of voltage regulation and damping enhancements [10]. A robust linear controller has been proposed to solve the problem of control action coordination due to variations in the generator terminal voltage and electric power [13]. A unified approach for a voltage regulator and PSS design based on predictive control in the $s$-domain is presented in Ref. [11].

Since the transient stability and voltage regulation are ascribed to different causes, some recent proposed scenarios apply a switching strategy of two different kinds of controller to cover different behaviours of system operation during transient and post-transient periods [14, 15]. The performance of these schemes essentially depends upon the selection of switching time. Moreover, the use of different control surfaces through a highly nonlinear structure increases the complexity of the designed controllers. Feedback linearisation schemes are also widely used in the design of robust controllers for power systems which linearise nonlinear power system models into a linear one. A feedback linearising controller (FBLC) is used to design a controller for a synchronous generator connected to an infinite bus in Ref. [16]. The direct feedback linearisation theory is easier to understand for power engineers, but it uses rotor angle and accelerating power as feedback signals and some additional sensors and differentiators are essential to measure these signals. Another type of FBLC to damp electromechanical oscillations in power systems is proposed in Ref. [17], but this is achieved at the expense of reducing the voltage regulation ability of the excitation system.

In Ref. [18], it is indicated that the implementation of an exciter control based on the FBLC theory requires a exact parameters of the power system which is usually not well defined in practice. Furthermore, the controllers designed through feedback linearisation require information of the power system topology, and the states must be measurable. In practice, it is very difficult to measure all the states of a power system. In addition, feedback linearisation schemes use rotor angle as an output function to cancel the inherent system nonlinearities. An exact FBLC is proposed in Ref. [19] for power systems with a dynamic loads where a dynamic load is connected to a SMIB system. From Ref. [19], it can be seen that the law is quite complex even for an SMIB system, and it would be more complex for a multimachine power system which is difficult to implement. To overcome the difficulty associated with the state variables, an outputfeedback linear quadratic Gaussian (LQG) excitation control scheme is proposed in Ref. [20]. The LQG controller is more realistic, because it is designed using only the measurable outputs and the state variables estimated from them. It is well known that the LQR controller provides good robustness in terms of gain margin and phase margin [21]. However, LQR and LQG controllers are unable to provide robustness against uncertainties with changes in operating conditions [22]. This motivates the use of robust LQG control techniques to ensure system stability for large disturbances.

The assessment of voltage stability in power systems has recently gained increasing attention, because voltage instability has been responsible for several major network collapses [23]. In contrast to the traditional machine instability, which deals with the rotor dynamics, voltage instability is closely related to the load behaviour [24]. Different studies have shown the importance of load representation in voltage stability analyses [23, 25]. Currently, although static load models are commonly used in the power industry to model dynamic behaviours of reactive loads, they do not adequately do so [26]. For any systems to be effective practically, the inclusion of dynamic load models is essential, but that representation exhibits even more nonlinear behaviours than the ones with constant impedance loads do. Therefore, dynamic load models are needed in order to analyse the system instability and to design controllers for enhancing transient stability along with voltage regulation.

In this article, we consider the problem of designing a linear controller for a nonlinear power system model, in 
such a way that this linear controller provides an acceptable performance over a wider operating region, as compared to other conventional linear controllers. The nonlinearities are dealt with by explicitly including the information about the system nonlinearities in the design formulation, using the Cauchy remainder of the Taylor series expansion $[27,28]$. The voltage controller and PSS are designed sequentially provided that the performance of one controller is not degraded by the other controller. The designed controllers significantly improve both the damping of electromechanical oscillations and voltage stability.

The article is organised as follows. In Section 2, the mathematical model of the power system devices under consideration is provided. The test system description and control task are presented in Section 3. Section 4 describes the linearisation technique and the process for obtaining the bounds on the nonlinear terms. Performance of the proposed new control design algorithm is demonstrated in Section 5. Finally, conclusions are drawn in Section 6.

\section{Power system model}

Under typical assumptions, the synchronous generator can be modelled by the following set of nonlinear differential equations [29]:

$$
\begin{gathered}
\dot{\delta}=\omega \omega_{s}-\omega_{s}, \\
\dot{\omega}=\frac{1}{2 H}\left[P_{m}-E_{q}^{\prime} I_{q}-D \omega\right], \\
\dot{E}_{q}^{\prime} \frac{1}{T_{d 0}^{\prime}}\left[E_{\mathrm{fd}}-E_{q}^{\prime}-\left(X_{d}-X_{d}^{\prime}\right) I_{d}\right],
\end{gathered}
$$

where $E_{\mathrm{fd}}$ is the equivalent emf in the exciter coil, $\delta$ is the power angle of the generator, $\omega$ is the rotor speed with respect to a synchronous reference, $E_{q}^{\prime}$ is the transient emf due to field flux linkage, $\omega_{S}$ is the absolute value of the synchronous speed in radians per second, $H$ is the inertia constant of the generator, $D$ is the damping constant of the generator, $T_{d o}^{\prime}$ is the direct-axis open-circuit transient time constant of the generator, $X_{d}$ is the synchronous reactance, $X_{d}^{\prime}$ is the transient reactance, and $I_{d}$ and $I_{q}$ are direct and quadrature axis components of stator current, respectively. The mechanical input power $P_{m}$ to the generator is assumed to be constant.

The excitation system is a high-gain static system, and terminal voltage is measured using a transducer with first-order dynamic:

$$
E_{\mathrm{fd}}=K_{a}\left(V_{c}+V_{p s s}\right), \quad \dot{V}_{t r}=\frac{1}{T_{r}}\left[-V_{t r}+V_{t}\right],
$$

where $V_{t r}$ and $T_{r}$ are the output and time constant of the voltage transducer, respectively, $K_{a}$ is the gain of the exciter amplifier, $V_{t}=\left[\left(E_{q}^{\prime}-X_{d}^{\prime} I_{d}\right)^{2}+\left(X_{d}^{\prime} I_{q}\right)^{2}\right]^{\frac{1}{2}}$ is the generator terminal voltage, and $V_{c}$ is the input to the exciter (output of the designed controller).

The output-feedback controller, shown in Figure 1, is represented as:

$$
\dot{\hat{x}}_{c}=A_{c} \hat{x}_{c}(t)+B_{c}\left(V_{\text {ref }}-V_{t r}\right), \quad V_{c}=C_{c} \hat{x}_{c}(t),
$$

where $A_{c}, B_{c}$, and $C_{c}$ are the appropriate matrices of the controller.

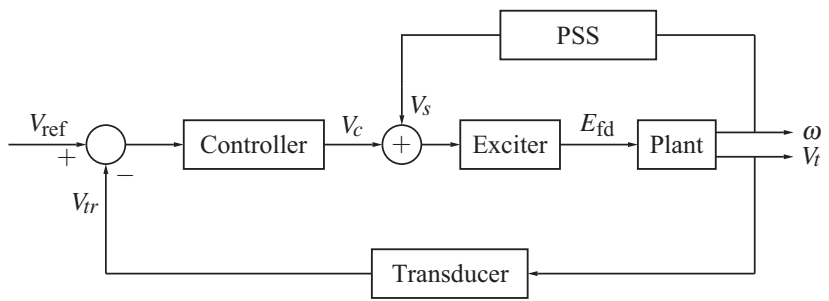

Figure 1 Excitation controller

A third-order model of an induction motor as presented in Ref. [4] has been used in this article. However, these equations represent the induction machine in its own direct and quadrature axes, which are different from the $d$ - and $q$-axes of the generator. A transformation is used to represent both dynamic elements with respect to the same reference frame as discussed in Ref. [4]. Then, the modified third-order induction machine model can be rewritten as:

$$
\begin{gathered}
\left(V_{d}+j V_{q}\right)=-\left(R_{s}+j X^{\prime}\right)\left(I_{d m}+j I_{q m}\right)+j E_{q m}^{\prime}, \\
\dot{s}=\frac{1}{2 H_{m}}\left[T_{m}-E_{m}^{\prime} I_{q m}\right], \\
\dot{E}_{m}^{\prime}=-\frac{1}{T_{d o m}^{\prime}}\left[E_{m}^{\prime}+\left(X-X^{\prime}\right) I_{d m}\right], \\
\dot{\delta}_{m}=s \omega_{s}-\omega_{s}-\frac{X-X^{\prime}}{T_{d o m}^{\prime} E_{m}^{\prime}} I_{q m} .
\end{gathered}
$$

The block diagram of the proposed controller is given in Figure 1. Speed and the terminal voltage of generator are used as the feedback signals. In this article, a coordinated PSS and voltage stability controller will be designed as suggested by the auxiliary input $V_{s}$ in Figure 1 . 


\section{Test system and control task}

One-line diagram of the 10 Machine New England system is shown in Figure 2. This system has been modified by adding a $25 \mathrm{~kW}$ induction motor at the terminal of each generator, and the modified system is used as a test system in this article. Generator 1 represents the aggregation of a large number of generators. The generation and total load in this system are 6,193.41 MW and 6,150.5 MW, respectively. The load in this article is modelled as (i) $50 \%$ induction motor load, (ii) 5\% transformer exciting current, (iii) $10 \%$ constant power, and (iv) 35\% constant impedance load.

The test system in this article, with 50\% dynamic load, has one unstable mode with a real eigenvalue at 0.45 and an undamped mode with eigenvalue of $-0.0091 \pm j 3.1$. Most significant normalized participation vectors for these two modes are shown in Table 1. The mode $-0.0091 \pm j 3.1$ is an electromechanical mode with a damping ratio of 0.0029 . The other mode with the eigenvalue 0.45 is a monotonic mode associated with the rotor electrical dynamics of generators and induction motors. This monotonic mode is introduced due to the replacement of constant impedance loads with induction motors. In this article, attention is directed to the design of robust control for these critical modes.
Table 1 Critical modes and participation factors

\begin{tabular}{llll}
\hline Modes & \multicolumn{3}{c}{ Participation factors } \\
\hline$-0.0091 \pm j 3.1$ & $\Delta \omega_{2}=1$ & $\Delta \delta_{2}=0.99$ & $\Delta \omega_{3}=0.26$ \\
0.45 & $\Delta E_{q 4}^{\prime}=1$ & $\Delta E_{q r_{4}}^{\prime}=0.78$ & $\Delta E_{d r_{4}}^{\prime}=0.71$ \\
\hline
\end{tabular}

The objective in the PSS design is to increase damping of the electromechanical mode by adding an auxiliary signal to the AVR. PSSs are designed to have very low gains in the frequency range outside of a narrow band centred around the resonant mode frequency. This necessitates the design of controllers to maintain system stability for other unstable or lightly damped modes. In this article, PSS is designed for $G_{2}$ and AVR for $G_{4}$ using locally measured signal.

\section{Linearisation and uncertainty modelling}

Conventionally, a linear controller is designed by neglecting the higher order terms of the Taylor series around an equilibrium point. In this article, we propose the use of a linearisation scheme which retains the contributions of the higher order terms in the form of the Cauchy remainder.

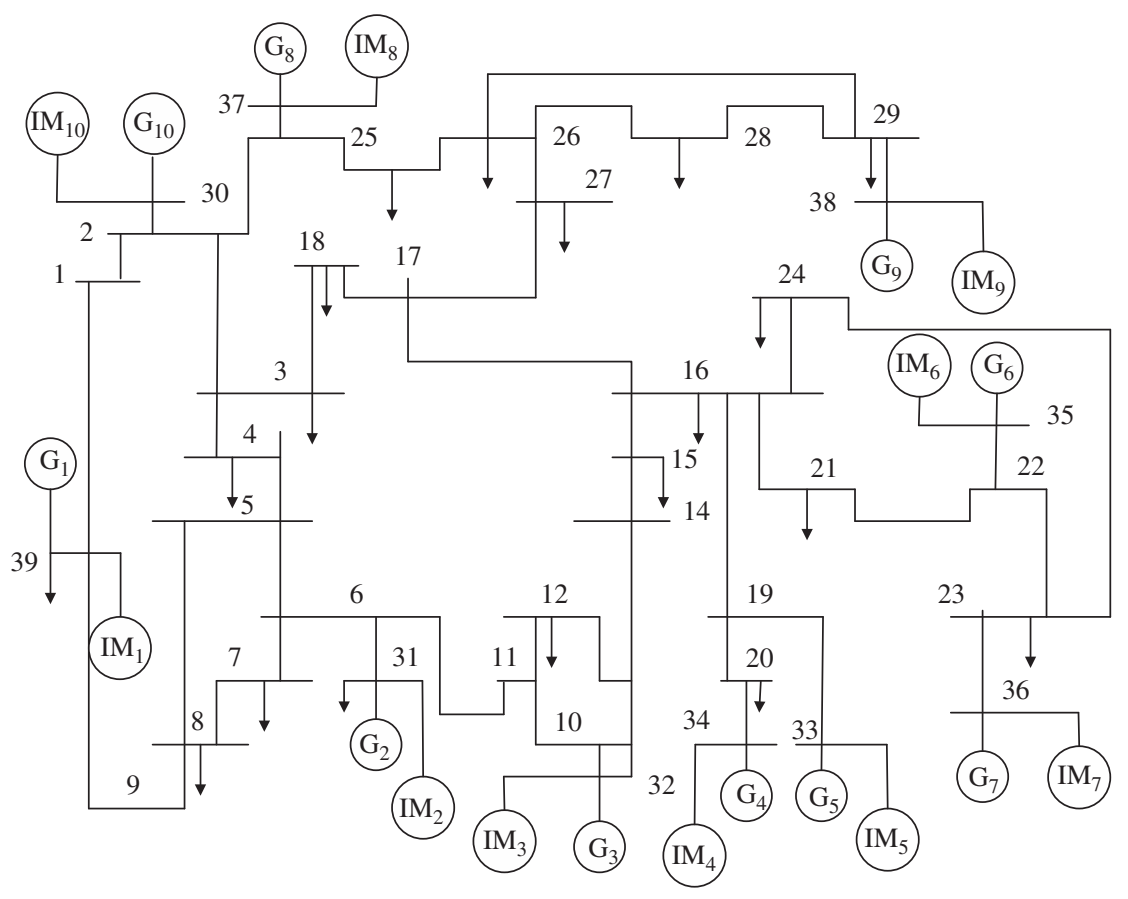

Figure 2 Test system 
The reformulation proposed in this article using Cauchy remainder allows us to represent the nonlinear power system models as:

$$
\begin{gathered}
\Delta \dot{x}(t)=A \Delta x(t)+B_{1} \Delta u(t)+B_{2} \xi(t), \\
\left.y(t)=C_{2} \Delta x(t)+D_{2} \xi(t)\right), \\
\zeta(t)=C_{1} \Delta x(t)
\end{gathered}
$$

where $\Delta x$ is the state vector, $\Delta u$ is the control input, $y(t)$ is the measured output, $\xi$ is known as the uncertainty input, and $\zeta$ is known as the uncertainty output. The procedure for obtaining the matrices in eqs (9)-(11) and bounding uncertainty has been described in the rest of this section.

Let $\left(x_{0}, u_{0}\right)$ be an arbitrary point in the control space; using the mean-value theorem, the test system dynamics can be rewritten as follows:

$$
\dot{x}=f\left(x_{0}, u_{0}\right)+L\left(x-x_{0}\right)+M\left(u-u_{0}\right),
$$

where

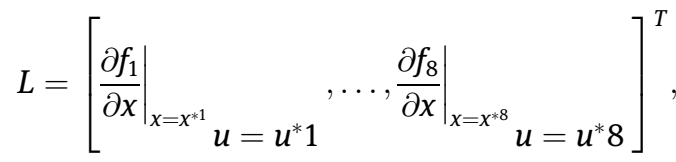

$$
\begin{aligned}
& M=\left[\left.\frac{\partial f_{1}}{\partial u}\right|_{x=x^{* 1}} u=u^{* 1} \quad, \ldots,\left.\frac{\partial f_{8}}{\partial u}\right|_{x=x^{* 8}} u=u^{*} 8\right]^{T}, \\
& f=\left[f_{1}, \ldots, f_{8}\right]^{T},
\end{aligned}
$$

where $\left(x^{* p}, u^{* p}\right), p=1, \ldots, 7$, denote points lying in the line segment connecting $(x, u)$ and $\left(x_{0}, u_{0}\right)$, and $f$ denotes the vector function on the right-hand side of the vector differential equations.

Letting $\left(x_{0}, u_{0}\right)$ be the equilibrium point and defining $\Delta x \triangleq x-x_{0}$ and $\Delta u \triangleq u-u_{0}$, it is possible to rewrite eq. (12) as follows

$$
\begin{aligned}
\Delta \dot{x} & =\dot{x}-\dot{x}_{0}, \\
& =A \Delta x+(L-A) \Delta x+\left(M-B_{1}\right) \Delta u+B_{1} \Delta u,
\end{aligned}
$$

where $A=\left.\frac{\partial f}{\partial x}\right|_{x=x_{0}} u=u_{0}, B_{1}=\left.\frac{\partial f}{\partial u}\right|_{x=x_{0}} u=u_{0}$. Eq. (13) is linear with respect to the control vector. Since $x^{* p}, p=1, \ldots, 7$, are not known, it is difficult to obtain the exact value of $(L-A)$, but it is possible to obtain a bound on $\|(L-A)\|$.

The system (13) is of the form which allows for an application of the minimax control design technique as presented in Ref. [30]. To apply this technique, we rewrite system (13) in terms of the block diagram shown in Figure 3.

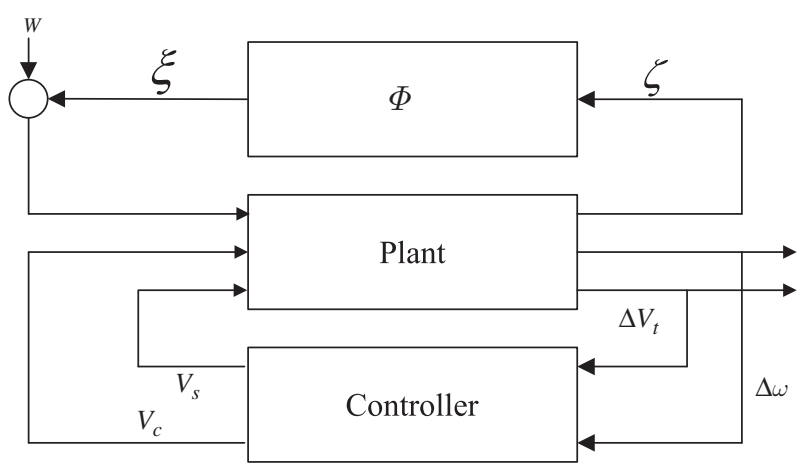

Figure 3 Control block diagram

In this figure, we introduce a fictitious signal $\xi$ such that

$$
(L-A) \Delta x=B_{2} \xi(t),
$$

where

$$
B_{2}=\operatorname{diag}\left(0, \frac{X_{d}-X_{d}^{\prime}}{T_{d o}^{\prime}}, \frac{1}{2 H}, \frac{1}{T_{r}}, \frac{X_{s}-X_{s}^{\prime}}{T_{d o m}^{\prime}}, \frac{1}{2 H_{m}}, \frac{X_{s}-X_{s}^{\prime}}{T_{d o m}^{\prime}}\right),
$$

and

$$
\xi=\tilde{\phi}(t) \tilde{C}_{1} \Delta x
$$

Matrix $\tilde{C}_{1}$ is chosen such that

$$
\begin{gathered}
\tilde{C}_{1}=\left[\begin{array}{ccccccc}
0 & 0 & 1 & 0 & 0 & 0 & 0 \\
0 & 0 & 0 & 0 & 1 & 0 & 0 \\
0 & 0 & 0 & 0 & 0 & 1 & 0 \\
0 & 0 & 0 & 0 & 0 & 0 & 1
\end{array}\right], \\
(L-A) \Delta x=B_{2} \tilde{\phi}(t) \tilde{C}_{1} \Delta x .
\end{gathered}
$$

The expressions for obtaining $\tilde{\phi}(t)$ can be determined in the same way as in Ref. [28]. The system can now be written as

$$
\Delta \dot{x}=A \Delta x+B_{1} \Delta u+B_{2} \xi(t) .
$$

In general, $x^{* p}, p=1, \ldots, 7$, are not known beforehand, it is difficult to obtain the exact value of $(L-A)$, but it is possible to obtain a bound on $\phi(t)$ shown in Figure 3. 
Next, we introduce a scaling parameter $\alpha$ and write $C_{1}=\sqrt{\alpha} \tilde{C}_{1}$, where $\alpha$ scales the magnitude of the uncertain output

$$
\zeta=\sqrt{\alpha}\left(\tilde{C}_{1} \Delta x\right) .
$$

We write $\phi(t)=\frac{1}{\sqrt{\alpha}}[\tilde{\phi}(t)]$. The value of $\alpha$ is chosen such that the uncertainty, $\phi(t)$, shown in Figure 3 satisfies,

$$
\|\phi(t)\|^{2} \leq 1
$$

From this, we have

$$
\|\xi(t)\|^{2} \leq \alpha\left\|\left(\tilde{C}_{1} \Delta x\right)\right\|^{2} .
$$

and we recover the norm bound constraints [30],

$$
\|\xi(t)\|^{2} \leq\|\zeta(t)\|^{2} .
$$

Condition (22) will enable us to apply the minimax LQG control design methodology to obtain a controller for the underlying nonlinear system. Robustness properties of the minimax LQG controller ensure that this controller stabilises the nonlinear system (9)-(11) for all instances of linearisation errors.

Associated with the uncertain system (9)-(11), we consider a cost functional $J$ of the form

$$
J=\lim _{T \rightarrow \infty} \frac{1}{2 T} E \int_{0}^{T}\left(\Delta x(t)^{T} R \Delta x(t)+\Delta u(t)^{T} G \Delta u(t)\right) d t,
$$

where $R \geq 0$ and $G>0, R \in R^{n \times n}, G \in R^{m \times m}$ and $E$ is the expectation operator.

The quadratic cost (23) is particularly suited to the design of an excitation controller for the power system. Every generator has an over-excitation limiter which limits the field voltage based on time integral of the voltage. This means that one can apply large voltages as long as they are for short duration. Thus, the quadratic cost optimisation is much more suitable in this situation as compared to $H_{\infty}$-norm based designs.

The minimax optimal control finds the controller which minimises $J$ over all admissible uncertainties. The cost function $J$ satisfies the following relationship [31]:

$$
\sup _{\|\xi\|^{2} \leq\|\zeta\|^{2}} J\left(u^{*}\right) \leq \inf _{\tau} V_{\tau}
$$

where $V_{\tau}$ is given by

$$
\begin{gathered}
V_{\tau}=\frac{1}{2} \operatorname{tr}\left[Y_{\infty} R_{\tau}+\left(Y_{\infty} C_{2}^{T}+B_{2} D_{2}^{T}\right)\left(D_{2} D_{2}^{T}\right)^{-1}\left(C_{2} Y_{\infty}+D_{2} B_{2}^{T}\right)\right. \\
\left.X_{\infty}\left(I-\frac{1}{\tau} Y_{\infty} X_{\infty}\right)^{-1}\right]
\end{gathered}
$$

$\tau$ is a free parameter and the matrices $X_{\infty}$ and $Y_{\infty}$ are the solutions to the following pair of parameter dependent algebraic Riccati equations [31]:

$$
\begin{aligned}
& \left(A-B_{2} D_{2}^{T}\left(D_{2} D_{2}^{T}\right)^{-1} C_{2}\right) Y_{\infty}+Y_{\infty}\left(A-B_{2} D_{2}^{T}\left(D_{2} D_{2}^{T}\right)^{-1} C_{2}\right)^{T} \\
& -Y_{\infty}\left(C_{2}^{T}\left(D_{2} D_{2}^{T}\right)^{-1} C_{2}-\frac{1}{\tau} R_{\tau}\right) Y_{\infty}+B_{2}\left(I-D_{2}^{T}\left(D_{2} D_{2}^{T}\right)^{-1} D_{2}\right) B_{2}^{T}=0,
\end{aligned}
$$

and

$$
\begin{aligned}
& X_{\infty}\left(A-B_{1} G_{\tau}^{-1} \gamma_{\tau}^{T}+\left(A-B_{1} G_{\tau}^{-1} \gamma_{\tau}^{T}\right) X_{\infty}\right. \\
& +\left(R_{\tau}-\gamma_{\tau} G_{\tau}^{-1} \gamma_{\tau}^{T}\right)-X_{\infty}\left(B_{1} G_{\tau}^{-1} B_{1}^{T}-\frac{1}{\tau} B_{2} B_{2}^{T}\right) X_{\infty}=0 .
\end{aligned}
$$

The solutions are required to satisfy the following conditions: $Y_{\infty}>0, X_{\infty}>0$, the spectral radius of the matrix $X_{\infty} Y_{\infty}$ is $\rho\left(X_{\infty} Y_{\infty}\right)<\tau, R_{\tau}-\gamma_{\tau}^{T} G_{\tau}^{-1} \gamma_{\tau} \geq 0, R_{\tau}=R+\tau C_{1}^{T} C_{1}$, $G_{\tau}=G+\tau D_{1}^{T} D_{1}$, and $\gamma_{\tau}=\tau C_{1}^{T} D_{1}$.

To obtain the minimax LQG controller, the parameter $\tau>0$ is chosen to minimise $V_{\tau}$. A line search is carried out to find the value of $\tau>0$ which attains the minimum value of the cost function $V_{\tau}$. This line search involves solving the Riccati equations (26) and (27) for different values of $\tau$ and finding the value which gives the smallest $V_{\tau}$.

The minimax LQG optimal controller is given by the equations:

$$
\begin{aligned}
\dot{\hat{x}}_{c}= & \left(A-B_{1} G_{\tau}^{-1} \gamma_{\tau}^{T}\right) \hat{x}_{c}-\left(\left(B_{1} G_{\tau}^{-1} B_{1}^{T}-\frac{1}{\tau} B_{2} B_{2}^{T}\right) X_{\infty}\right) \hat{x}_{c} \\
& +\left(I-\frac{1}{\tau} Y_{\infty} X_{\infty}\right)^{-1} \\
& \left(Y_{\infty} C_{2}^{T}+B_{2} D_{2}^{T}\right) \times\left(D_{2} D_{2}^{T}\right)^{-1}\left(y-\left(C_{2}+\frac{1}{\tau} D_{2} B_{2}^{T} X_{\infty}\right) \hat{x}_{c}\right),
\end{aligned}
$$

$$
u=-G_{\tau}^{-1}\left(B_{1}^{T} X_{\infty}+\gamma_{\tau}^{T}\right) \hat{x}_{c}
$$

The output matrix for the voltage controller is defined as $C_{2}=[0,0,0,1,0,0,0]^{T}$. The theory requires that $D_{2} D_{2}^{\prime}>0$ as discussed in Ref. [30]. This property is required by the design procedure, but it does not represent any physical characteristics of the system, so we choose the value of $D_{2}$ as small as possible. Eqs (9)-(11) provide a new representation of the power system model which contains the linear part and also another part with higher order terms. The new formulation presented in this section is used with the minimax LQG control theory to design the excitation controllers for the generator as in Refs [4, 30]. 


\section{Controller design and performance evaluation}

Prior to the controller design, we carry out several large disturbance simulations to get an idea of the region of interest. The maximum value of $\phi(t)$ is obtained over this region and not globally. If the maximum value of $\phi(t)$ is evaluated over the entire uncertainty region, the calculation burden will be very high, and it will lead to a conservative controller. The controller is then designed as follows:

Step 1. From the simulations of the faulted system, obtain the range of the variation of all state variable and form a polytope $\Omega$ with corner points given by $\left(x_{0_{p}}-x_{f_{p}}\right)$ and $\left(x_{f_{p}}+x_{0_{p}}\right), p=1, \ldots, 7$, where $x_{f_{p}}$ is the largest variation of the $p$ th state variable about its equilibrium value $x_{0_{p}}$. Formally, $x \in \Omega$ if $\left|x-x_{0_{p}}\right| \leq\left|x_{f_{p}}-x_{0_{p}}\right|$.

Step 2. Obtain

$$
\alpha^{*}=\max _{\chi^{* p} \in \Omega}\left\{\alpha:\|\phi(t)\|^{2}<1\right\} .
$$

The process to obtain $\alpha^{*}$ involves obtaining the maximum value of $\|\tilde{\phi}(t)\|$ over the polytope $\Omega$.

Step 3. Check if there exists a feasible controller with $\alpha=\alpha^{*}$.

Step 4. If we obtain a feasible controller in the above step, either enlarge the polytope $\Omega$, i.e. increase the operating region of the controller, or if we have arrived at the largest possible polytope then perform an optimal search over the scalar parameter $\tau$ to get the infimum of cost function [4]. If there is no feasible solution with the chosen $\alpha=\alpha^{*}$, reduce the polytope, $\Omega$ and go to Step 2 .

For the given power system model, we are able to obtain a feasible controller with the value of $\alpha=0.925$. The controller is stabilising for all variations of states in the polytope region $\Omega$ formed by corner points $\left[\bar{\delta}, \bar{\omega}, \bar{E}_{q}^{\prime}, \bar{V}_{t r}, \bar{\delta}_{m}, \bar{s}, \bar{E}_{m}^{\prime}\right]$ and $\left[\underline{\delta}, \underline{\omega}, \underline{E}_{q}^{\prime}, V_{t r}, \underline{\delta}_{m}, s, E_{m}^{\prime}\right]$ with the values: $\left|\bar{\delta}+\delta_{0}\right|=45^{\circ}$, $\left|\underline{\delta}-\delta_{0}\right|=45^{\circ},\left|\bar{\omega}+\omega_{0}\right|=0.375 \mathrm{pu},\left|\underline{\omega}-\omega_{0}\right|=0.375 \mathrm{pu}$,

$\left|\bar{E}_{q}^{\prime}+E_{q_{0}}^{\prime}\right|=0.435, \quad\left|E_{q}^{\prime}-E_{q_{0}}^{\prime}\right|=0.435, \quad \bar{V}_{t r}=V_{t r 0}+0.275$

$\mathrm{pu}, \quad V_{t r}=V_{t r 0}-0.275 \quad \mathrm{pu}, \quad \bar{\delta}_{m}=\delta_{m 0}+41.19^{\circ}$, $\underline{\delta}_{m}=\delta_{m 0}-41.19^{\circ}, \bar{s}=s_{0}+0.225 \mathrm{pu}, s=s_{0}-0.225 \mathrm{pu}$, $\bar{E}_{m}^{\prime}=E_{m 0}+0.25 \mathrm{pu}$, and $E_{m}^{\prime}=E_{m 0}-0.25 \mathrm{pu}$. Although the designed controller is not globally stabilising, we can be confident that it will stabilise the system for most contingencies.

In this article, the PSS as shown in Figure 4 has been designed using the standard technique which uses the change in speed $\Delta \omega$ as the feedback variable. PSS was designed subsequent to the design of voltage controller. The PSS parameters are $T_{w}=5, K_{\mathrm{STAB}}=0.43, T_{1}=0.25$, $T_{2}=0.02, T_{3}=0.0 .252$, and $T_{4}=0.039$.

\subsection{Controller performance evaluation}

As mentioned earlier, the voltage controller and PSS have been designed sequentially. At first, voltage controller is designed, and then the PSS is designed including voltage controller. The objective of PSS design is to damp electromechanical oscillations in power systems. However, this should not be done at the expense of reducing the voltage regulation ability of the excitation system. Using simulation results given below, we show that the PSS does not have an adverse effect on the voltage controller.

A simulation is carried out by applying a symmetrical three phase to ground short circuit fault on the middle of the line 16-19. The fault is cleared after $150 \mathrm{~ms}$. Figures 5 and 6 show the terminal voltage and real power output of generator $G_{4}$ with both voltage controller plus PSS and only the voltage controller due to the three-phase fault on the middle of the line 16-19. Figure 5 shows that the PSS improves the voltage response. This is due to the improved damping of the electromechanical modes, which is also visible from Figure 6.

Responses of terminal voltage and reactive power output of generator $G_{2}$, when a two-line to ground fault (2LG) occurs on the middle of the line 5-6 from phase $B$ and phase $\mathrm{C}$ to ground, are shown in Figures 7 and 8, respectively. From Figures 7 and 8, it can be seen that the

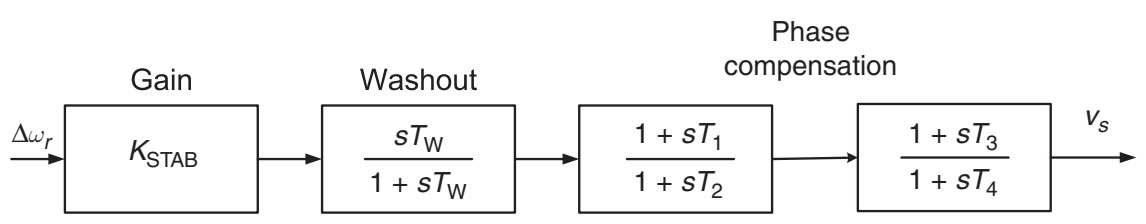

Figure 4 PSS block diagram 


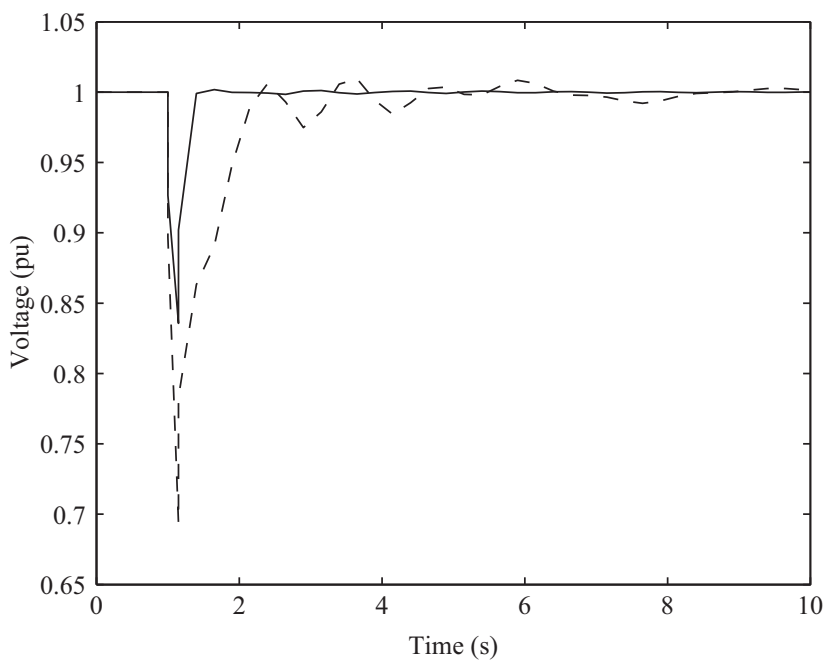

Figure 5 Terminal voltage $\left(G_{4}\right)$ for three-phase fault in the middle of line 16-19. (Solid line voltage controller + PSS and dashed line voltage controller only)

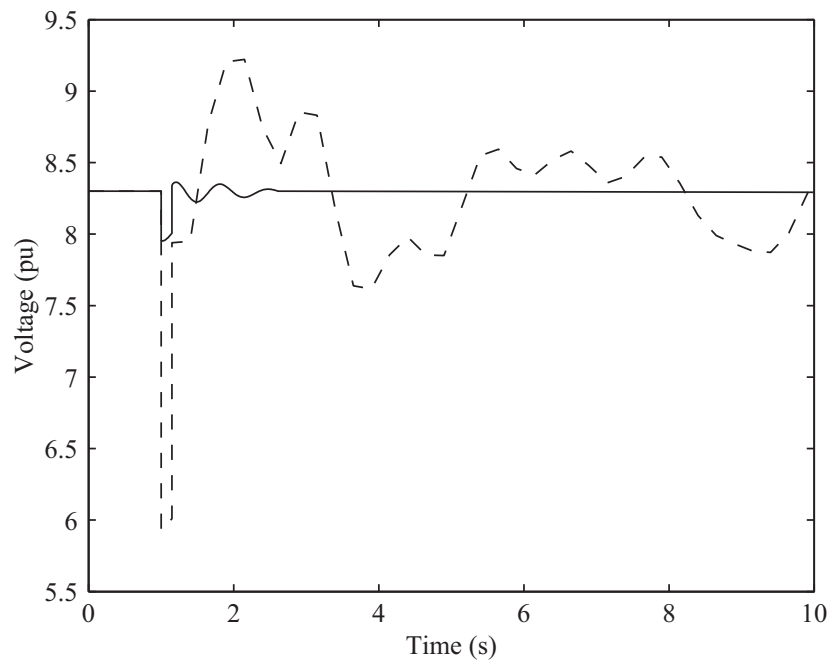

Figure 6 Real power output $\left(G_{4}\right)$ for three-phase fault in the middle of line 16-19. (Solid line voltage controller + PSS and dashed line voltage controller only)

performance of the voltage controller is not much affected by the PSS in stabilising the voltage and producing reactive power output of generator. From these figures, it is clear that the designed controllers also stabilise the generators under unsymmetrical faults.

In the preceding part of the article, the voltage controller is designed first and then the PSS - Design A. This order of the controller design is compared with the design where the PSS is designed first and then the voltage controller - Design B. The closed-loop dominant modes

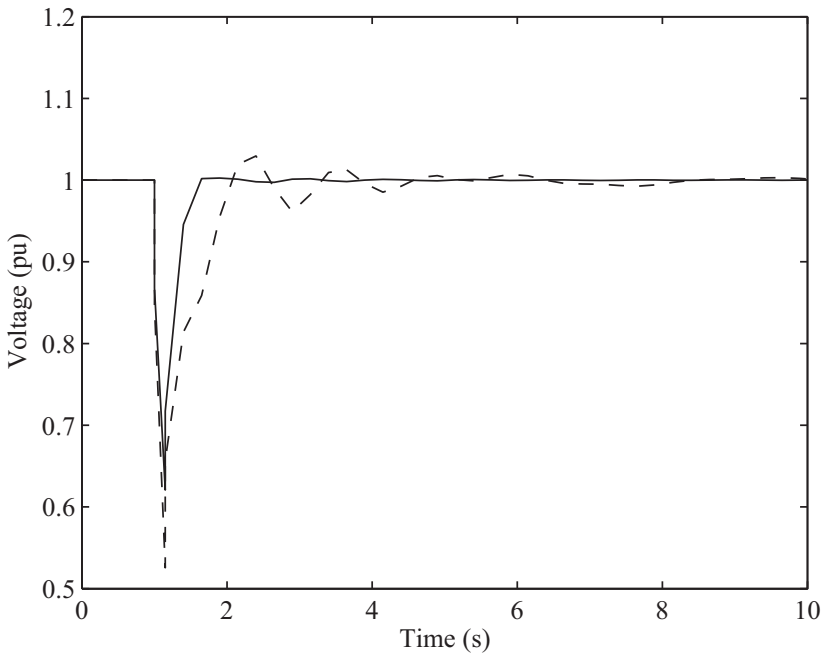

Figure 7 Terminal voltage $\left(G_{2}\right)$ for the unsymmetrical fault in the middle of line 5-6. (Solid line voltage controller + PSS and dashed line voltage controller only)

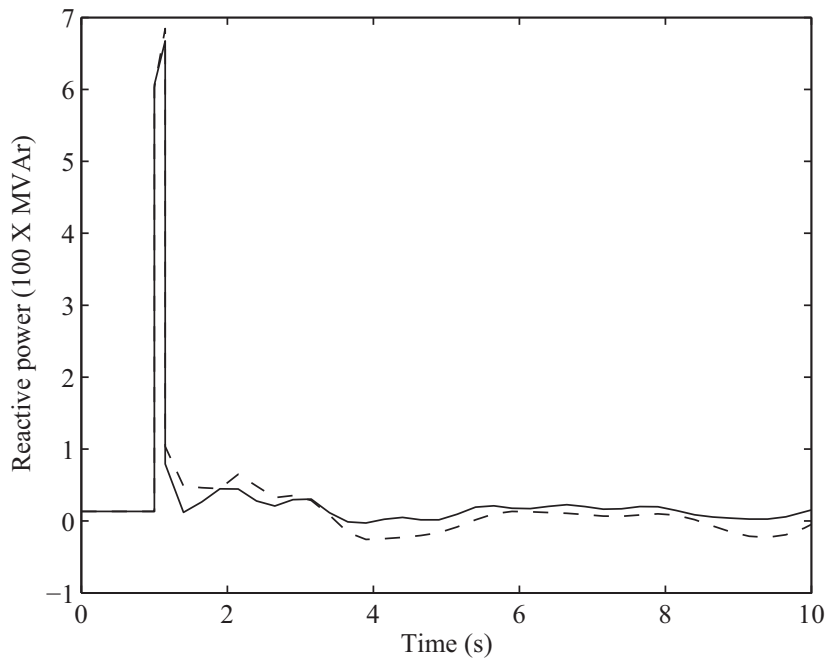

Figure 8 Reactive power output $\left(G_{2}\right)$ for the the unsymmetrical fault in the middle of line 5-6. (Solid line voltage controller + PSS and dashed line voltage controller only)

are, Design A: $-0.51 \pm j 0.326$ and with Design B: $-0.0091 \pm j 3.04$. The closed-loop behaviour is also compared for a $20 \%$ increase in load at bus 20 . The terminal voltage and real power at generator $G_{4}$ is shown in Figures 9 and 10. With Design B, the terminal voltage dips to $\sim 0.75 \mathrm{pu}$, and the real power oscillates for well over $20 \mathrm{~s}$ with a peak of $670 \mathrm{MW}$. The response for Design A is well damped and both terminal voltage and real power stay within a narrow and acceptable range. This result can be explained by the fact that in Design $B$, the 


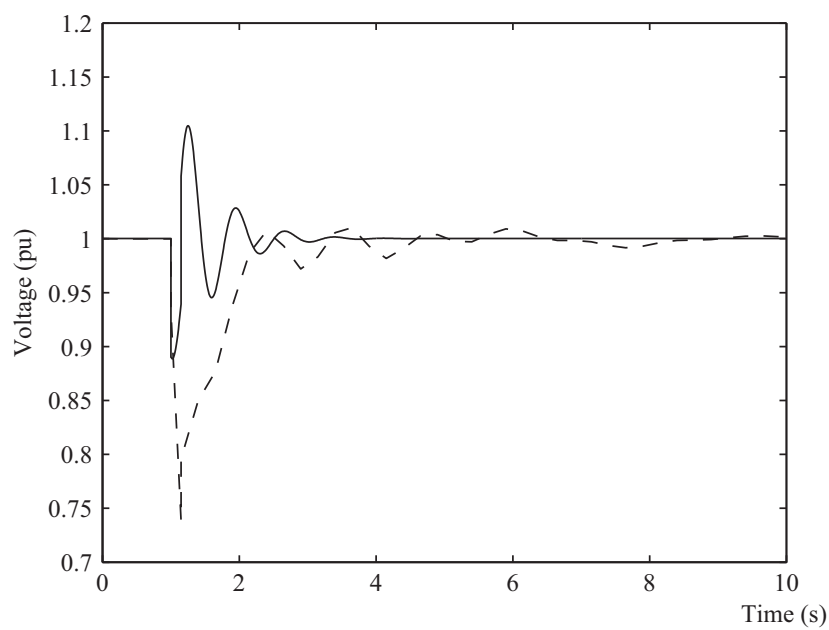

Figure 9 Terminal voltage $\left(G_{4}\right)$ for $20 \%$ change in load at bus 20 . (Solid line controllers with Design A and dashed line Design B)

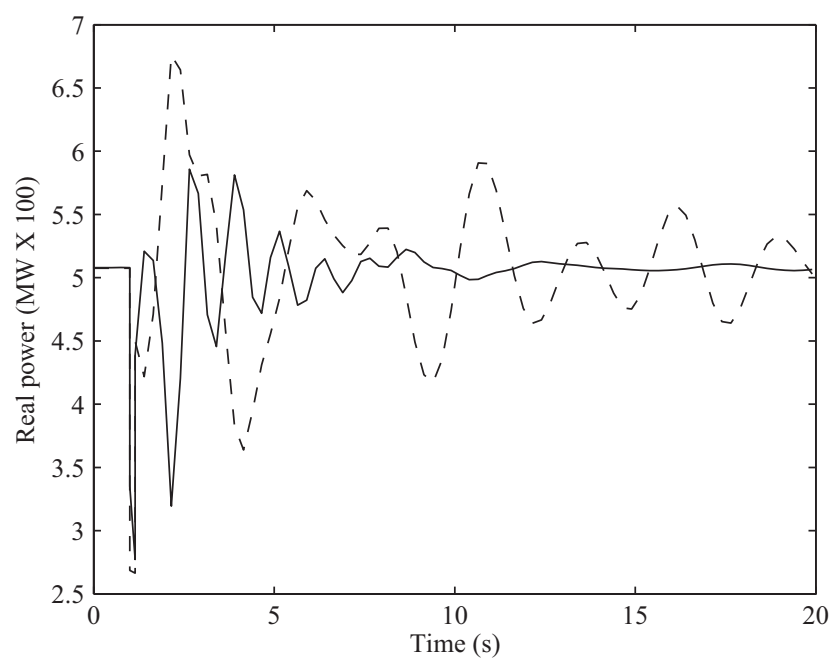

Figure 10 Real power output $\left(G_{4}\right)$ for $20 \%$ change in load at bus 20. (Solid line controllers with Design A and dashed line Design B)

voltage controller changes the required phase lead to be provided by the PSS.

The performance of the designed controllers are also compared with the performance of the conventional IEEE AC1A exciter and IEEEST stabiliser. Here, a simulation is performed for a severe symmetrical three-phase fault at bus 20 . The fault is cleared after $0.15 \mathrm{~s}$. Figures 11 and 12 show terminal voltage and angle response of the generators $G_{4}$ and $G_{2}$, respectively. From Figures 11 and 12, we can see that the proposed controller stabilises voltage within few cycles of fault occurrence and damps out the power angle oscillations. It is clear that the proposed controller has a better performance in terms of settling time, damping, overshoot, and oscillations.

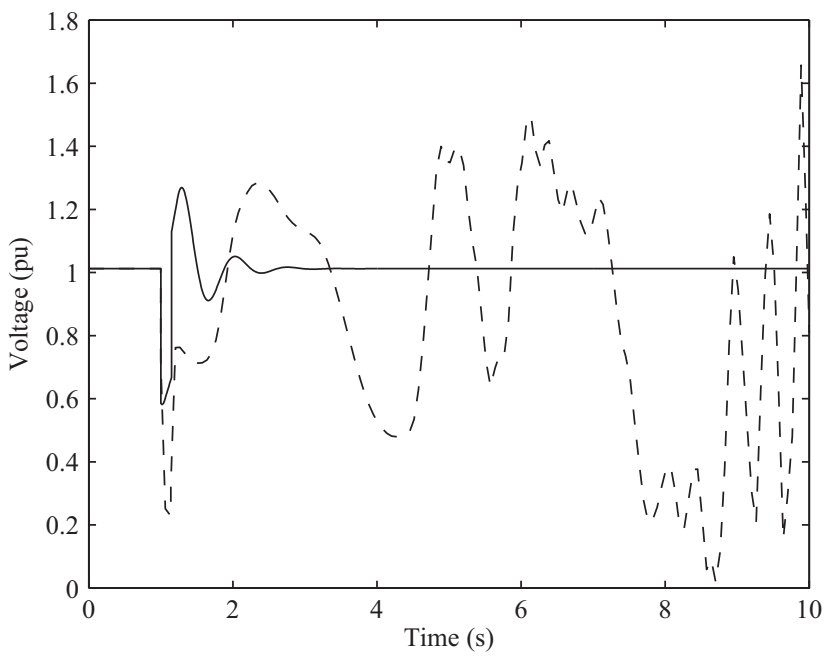

Figure 11 Terminal voltage $\left(G_{4}\right)$ for the three-phase fault. (Solid line designed controllers and dashed line AC1A exciter and IEEEST stabiliser)

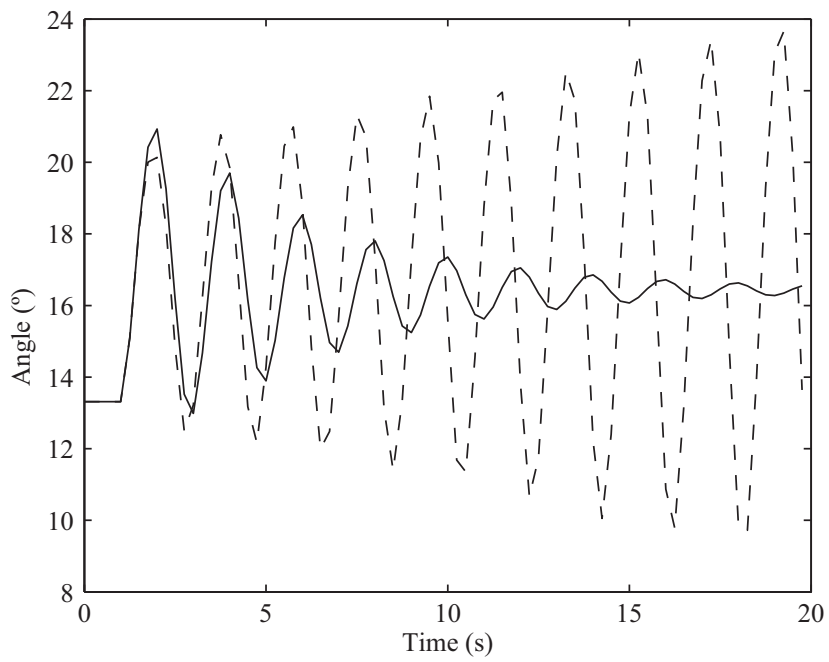

Figure 12 Angle response $\left(G_{2}\right)$ for the three-phase fault. (Solid line designed controllers and dashed line AC1A exciter and IEEEST stabiliser)

\section{Conclusions}

The results demonstrate that robust controller designed in this article, with a proper bounding of the uncertainty due to unmodelled nonlinear dynamics, performs well over a large region of operation. From this work, it can be concluded that the conservativeness of robust control can be overcome with the proposed method for bounding the unmodelled dynamics. The bounding method relies on the special dynamic behaviour of power systems 
which favours the evolution of the dynamics about certain trajectories. The quadratic objective function is a good choice to damp the poles of the closed-loop system. The chosen robust control method, minimax LQG, is able to design high performing controllers with output

\section{References}

1. Pariz N, Shanechi HM, Vaahedi E. Explaining and validating stressed power systems behavior using modal series. IEEE Trans Power Syst 2003;18:778-85.

2. Kundur P, Paserba J, Ajjarapu V, Andersson G, Bose A, Canizares C, et al. Definition and classification of power system stability. IEEE Trans Power Syst 2004;19:1387-401.

3. Zhou X, Yi J, Song R, Yang X, Li Y, Tang H. An overview of power transmission systems in china. Energy 2010;35:4302-12.

4. Hossain MJ, Pota HR, Ugrinovskii V, Ramos RA. Voltage mode stabilisation in power systems with dynamic loads. Int J Electrical Power Energy Syst 2010;32:911-20.

5. Schaefer RC, Kim K. Power system stabilizer performance with summing point type var/power factor controllers. In Conference Record of the 2006 IEEE IAS Pulp and Paper Conference, 2006:1-7.

6. Leon JA, Taylor CW. Understanding and solving short term voltage stability problems. In IEEE Power Engineering Society Summer Meeting, Chicago, IL, 2002:745-52.

7. Leona AE, Solsonaa JA, Figueroaa JL, Valla MI. Optimization with constraints for excitation control in synchronous generators. Energy 2011;36:5366-73.

8. Bevrani H, Hiyama T. Stability and voltage regulation enhancement using an optimal gain vector. In IEEE Power Engineering Society General Meeting, Canada, 2006:1-6.

9. Bevrani H, Hiyama T. Robust coordinated AVR-PSS design using $H_{\infty}$ static output feedback control. IEEJ Trans Power Energy 2007;127:70-6.

10. Lawt KT, Hill DJ, Godfrey NR. Robust co-ordinated AVR-PSS design. IEEE Trans Power Syst 1994;9:1218-25.

11. Saidy M. $\underline{A}$ unified approach to voltage regulator and power system stabiliser design based on predictive control in analogue form. Int J Electrical Power Energy Syst 1997;19:103-09.

12. Cao YJ, Jiang L, Cheng SJ, Chen DD, Malik OP, Hope GS. A nonlinear variable structure stabilizer for power system stability. IEEE Trans Energy Conversion 1994;9:489-95.

13. Heniche A, Bourles H, Houry MP. A desensitized controller for voltage regulation of power systems. IEEE Trans Power Syst 1995;10:1461-5.

14. Guo Y, Hill DJ, Wang Y. Global transient stability and voltage regulation for power systems. IEEE Trans Power Syst 2001;16:678-88.

15. Yadaiah N, Kumar AG, Bhattacharya JL. Fuzzy based coordinated controller for power system stability and voltage regulation. Electric Power Syst Res 2004;69:169-77.

16. Mahmud MA, Pota HR, Hossain MJ. Full-order nonlinear observer-based excitation controller design for interconnected feedback which is a major consideration for practical systems. The computational burden in the uncertainty bounding and controller design is not of concern as demonstrated by the design of the controller for the tenmachine New England system. power systems via exact linearization approach. Int J Electrical Power Energy Syst 2012;41:54-62.

17. Ramos RA, Alberto LF, Bretas NG. Linear matrix inequality based controller design with feedback linearisation: application to power systems. IEE Proc Control Theory Appl 2003;150:551-6.

18. Chapman JW, Ilic MD, King CA. Stabilizing a multi-machine power system via decentralized feedback linearizing excitation control. IEEE Trans Power Syst 1993;8:830-8.

19. Mahmud MA, Hossain MJ, Pota HR. Nonlinear excitation control of power systems with dynamic loads via feedback linearization. In 20th Australasian Universities Power Engineering Conference (AUPEC), 2010:1-6.

20. Seo JC, Kim TH, Park JK, Moon SI. An LQG based PSS design for controlling the SSR in power systems with series-compensated lines. IEEE Trans Energy Conversion 1996;11:423-8.

21. Anderson B, Moore JB. Optimal control: linear quadratic methods. USA: Prentice-Hall, 1990.

22. Ray LR. Stability robustness of uncertain LQG/LTR systems. IEEE Trans Automatic Control 1993;38:304-8.

23. Taylor CW. Power system voltage stability. New York: McGrawHill, 1994.

24. Vu KT, Liu CC, Taylor CW, Jimma KM. Voltage instability: mechanism and control strategies [power systems]. Proc IEEE 1995;83:1442-55.

25. Overbye TJ. Effects of load modelling on analysis of power system voltage stability. IEEE Trans Power Syst 1994;16:329-38.

26. Li Y, Chiang H-D, Choi B-K, Chen Y-T, Lauby D-H. Load models for modeling dynamic behaviors of reactive loads: evaluation and comparison. Int J Electrical Power Energy Syst 2008;30:497-503.

27. Hossain MJ, Pota HR, Ugrinovskii V, Ramos RA. A novel STATCOM control to augment LVRT capability of fixed-speed induction generators. In 48th IEEE Conference on Decision and Control, Shanghai, China, 2009:7843-8.

28. Hossain MJ, Pota HR, Ugrinovskii V, Ramos RA. Simultaneous STATCOM and pitch angle controls for improved LVRT capability of fixed-speed wind turbines. IEEE Trans Sustainable Energy 2010;1:142-51.

29. Hossain MJ, Pota HR, Ugrinovski V. Short and long-term dynamic voltage instability. In 17th IFAC World Congress, Seoul, Korea, 2008:9392-7.

30. Ugrinovskii VA, Petersen IR. Minimax LQG control of stochastic partially observed uncertain systems. SIAM J Control Optimization 2001;40:1189-226.

31. Petersen IR, Ugrinovskii VA, Savkin AV. Robust control design using $\mathrm{H}_{\infty}$ methods. London: Springer, 2000. 